\title{
An Enhanced Hybrid Item Recommender Model for Nigerian Online Stores
}

\author{
A.T. Olaniran \\ Obafemi Awolowo \\ University \\ Ile-Ife, Nigeria
}

\author{
I.O. Awoyelu \\ Obafemi Awolowo \\ University \\ Ile-Ife, Nigeria
}

\author{
A.O. Amoo \\ Obafemi Awolowo \\ University \\ lle-Ife, Nigeria
}

\author{
B.O. Akinyemi \\ Obafemi Awolowo \\ University \\ lle-Ife, Nigeria
}

\author{
R.O. Abimbola \\ Obafemi Awolowo \\ University \\ lle-Ife, Nigeria
}

\begin{abstract}
Item recommendation is the process of recommending goods sold on online stores to visitors and existing customers of the store to aid their shopping transactions processes. Majority of the online stores in Nigeria have their shopping systems implemented similar to foreign online stores' templates. Adapting these foreign shopping system templates to meeting the needs of Nigerian consumers has been quite challenging. This is due to the unavailability and sparsity of ratings needed by the systems for the generation of these recommendations, thus Nigerian online stores focus on the provision of nonpersonalized recommendations. The peculiarities of Nigerian consumers call for the provision of personalized item recommendations using alternative information other than ratings information. A hybrid item recommender system that has been demographically enhanced is being proposed in this paper. The model was formulated using the search method, user profiling and association rule mining for the contentbased item recommendations. The vector similarity and the adjusted cosine similarity methods were used for formulating the collaborative item recommendations. The demographic item recommendations were then formulated using the clustering and association rule methods.
\end{abstract}

The performance of the system was evaluated using Mean Absolute Error (MAE) and Root Mean Square Error (RMSE). The results of the performance evaluation carried out on the system showed that the system was able to reduce the Mean Absolute Error of the existing system by $61.24 \%$ and the Root Mean Square Error by $37.23 \%$ in content-based recommendations. In collaborative recommendations, evaluation results further showed that the new system was able to reduce the Mean Absolute Error of the existing system by $63.16 \%$ and the Root Mean Square Error by $39.30 \%$.

\section{General Terms}

Information Filtering and Access, Recommender Systems

\section{Keywords}

Purchase data, Demographic data, Item recommendation, Online Shopping

\section{INTRODUCTION}

One of the most successful applications in online shopping is 'personalized recommendation services' [17]. Recommender systems (RS) are software tools and techniques for providing suggestions to a user of a system [10]. They have become fundamental applications in electronic commerce and information access. They are widely used on online stores for effectively pruning through large customer and system data in order to generate recommendation of items that best meet user needs and preferences.
These systems have been grouped into personalized and nonpersonalized RS [4] and [12]. Most online stores have a recommendation engine embedded in their systems which provides personalized and non-personalized recommendations on the stores. This is why such stores can be regarded as online shopping recommender systems.

Personalized recommendations are targeted towards meeting the individual needs of the users of a recommender system. They are aimed at supporting shoppers in their various decision making processes while carrying out shopping activities online and are generated using ratings of items on the online store. They are also sometimes generated using the demographics of shoppers, mostly for shoppers who lack sufficient ratings that can be used by the system to provide them recommendations.

Non-personalized recommendations are targeted towards meeting the collective needs of all users of a recommender system. They are generated based on what other customers have said about certain products/items (product reviews), the top rated retailers on the store, items favourited by shoppers, analysis of shoppers' past buying behaviours etc. They are often presented as general advertisements on the store's homepage, discounts, offers, coupons etc.

Majority of the Nigerian online stores provide nonpersonalized item recommendations to their shoppers due to the challenges associated with the provision of personalized item recommendations. Nigerian retailers (online store owners), thus, embrace the provision of non-personalized item recommendations as they are much easier to generate and they also require little or no efforts from shoppers. They are also easy to implement on the stores as they are often automated.

A deep understanding and knowledge of the behaviour, the needs and the expectations of consumers according to [5], is required for the development of an online store that is suited to meet the special needs of a particular group of consumers. The peculiarities of Nigerian consumers, the Nigerian retail market and the Nigerian business environment are the factors considered in proposing a hybrid item recommender model as demonstrated in this paper.

\section{EXISTING WORKS}

A number of works have been carried out on the subject of RS. These systems have been used in the recommendation of web pages, new articles, documents, expertise, and items sold on online stores. Some of these works has been reviewed and critiqued in this paper. This is to provide an understanding of how the model proposed in this paper intends to address the lags in these works.

As most recommender systems use the collaborative or 
content-based filtering techniques or a combination of both to predict new items of interest for its users, more recent recommender systems are now combining demographic filtering with the earlier filtering techniques in developing hybrid recommender systems. This is to enhance the recommendation processes of the earlier techniques. Many of these filtering techniques when used in developing recommender systems individually have their own advantages and disadvantages. However, when combined into hybrids to make up for the disadvantages of the individual techniques, they perform quite well. Nonetheless, these hybrids still encounter various problems either with their recommendation processes or with the quality of the recommendations they provide.

Many scholars who have worked on online recommender systems have proposed different methods to addressing the various problems being encountered by these systems, some of which are the cold-start problem, the new-user and newitem problems, scalability problems, information overloading problems and others. Various propositions have been made such as enhancing the quality of hybrid recommender systems' recommendations using a standard similarity metrics to find user and item similarities, using dimensionality reduction techniques to scale through large data sets, introduction of demographic filtering by obtaining demographic information to characterize users and find similarities between them, using a standard demographic vector such as age for finding user similarities and enhancing the collaborative and content-based filtering techniques with other techniques.

[6] addressed scalability concerns in user-based collaborative recommendation systems by analyzing the user-item matrices in other to generate recommendations without the algorithm spanning through customer data on the system. Even though they were able to show that conditional probability-based item similarity scheme and higher-order item-based models provide reasonably accurate recommendations; they however did not consider the reduction of customer data stored on the system so as to reduce the data the algorithm spans through as will be done in this paper.

[16], on the other hand, addressed the non-utilization of demographic information in developing recommender systems by proposing enhancing collaborative filtering algorithms using singular value decomposition (SVD) as an augmentation technique and demographic data as an additional source of information to improve recommendation quality through the formation of demographic correlations for finding similarities in user and item neighbourhoods. They however did not consider the utilization of demographic data for the generation and provision of demographic recommendations to users of recommender systems as it is being done in this paper.

[2] addressed the cold-start problem (sparse/no ratings) of document recommender systems by computing user similarities using a collaborative filtering algorithm that generates recommendations based on past ratings of items and the demographic vector age. They thus generated a new similarity measure by combining results for recommending documents. This reduced user efforts through ratings as no initial ratings by new users was required by the system for recommendation generation. Even though they enhanced the collaborative filtering process using demographic vector age, they however did not consider using the demographic vector for finding similarities for all users of the system and for recommendation generation as it is being considered in this paper.

[1] addressed the problem of finding a standard metric to measure user similarities for users of recommender systems in finding similar users for new users of the system. They formulated a standard similarity metric by using genetic algorithms to assign values and weights to users who have similar pattern of ratings through a linear combination of values and weights. This was to find similarities for users with sparse or no ratings. Even though they were able to improve the prediction quality and performance of the system, they however did not explore using vector similarity methods for finding user similarities as presented in the proposed model.

[8] reviewed the various steps undertaken in user profiling and personalization and modeled user needs for personalization in recommender systems. They proposed generating recommendations through the calculation of item similarities with user profiles using the vector space model for generation of users 'points of interest' i.e. items often considered. Modeling the collective needs of users using their points of interest does not give room for modeling the individual needs of users as will be done in the proposed model.

[3] addressed the cold-start/new-user problems of recommender systems by proposing using demographics age, gender, race, disabilities, educational attainment, home ownership employment and occupation for finding user similarities using a neighbouring technique to form user clusters. They were able to achieve accuracy and high coverage in the system's recommendation and their algorithm outperformed conventional filtering algorithms as well as naïve methods. They however failed to consider limiting the number of demographic characteristics that will be utilized by the system due to the problems associated with obtaining demographic information from users as the provision of demographic information on a system can be sometimes considered invasive by users.

[14] also addressed cold-start problems in web page recommender systems by examining various information that can be used to determine if web pages can be recommended. They used demographic attributes for finding similarities between users with similar ratings. They were able to generate recommendations for users with sparse ratings, but did not specify the demographic attributes used.

[9] analyzed some of the problems and challenges encountered in deploying recommender systems by mining web browsing patterns for generating most visited web pages by users so as to present them as recommendations. However, mining web access records alone for recommendations generating cannot be used in modeling the individual needs of users of a recommender system accurately. This paper explores alternative information for modeling user needs and recommendation generation.

[11] developed an academic expertise recommender system to address the problem of finding field experts for academic collaborations. The system was able to find experts for possible research collaborations amongst those registered on the system. They system was however a hybrid of the contentbased and collaborative recommendation approaches. In this paper, the system incorporates three approaches to recommendation generation for developing a hybrid model. 
[13] addressed the challenges of building hybrid recommender systems that use linked open ratings data from different databases. A hybrid multi-strategy approach was used to combine the recommendation results of different base and generic recommenders using stacking regression and rank (ratings) aggregation for producing a final recommendation. Even though they were able to generated ratings popularity scores for recommendation generation, they however failed to explore the use of demographic recommenders as will be considered in the proposed model.

[7] predicted user preferences in a group context by evaluation the model in their earlier work where a multifaceted hybrid recommender system i.e. a recommender system that accepts and integrates different sets of data inputs.Even though they were able to analyze the impact of demographic data in predicting user needs in a group context, they did not predict the needs of users individually as will be considered in the proposed model.

[15] proposed a standard architectural framework, the Semantic Enhanced Personalizer (SEP) to integrate three recommendation techniques, the original, semantic and category-based techniques for enhancing the content-based and collaborative recommendation processes in document recommendation. The framework accomplishes the user-based and item-based approaches of recommendations and also overcomes the cold-start and sparsity problems using three recommendation modules, the original, semantic and category-based recommendation modules. Original recommendations were based on the content-based and collaborative recommendation approaches and also on contextual information and document ratings provided by the users of the system. Semantic recommendations were performed using various data mining techniques such as clustering, association-rule-mining and similarity measures.

For category-based recommendations, frequent keywords were extracted from visited document URLs and strong association rules were formed based on the keywords. Their framework avoids the need for ratings information in recommendation generation. It however presents the challenge of not being able to provide personalized recommendations to users of the system. This is because mining documents URLs visited by users collectively cannot be sufficient to model the individual needs of the users of the system. Each user has needs and preferences that the system must cater for and the SEP framework cannot attend to this.

In an online store scenario as it is being considered in this paper, shoppers are more likely going to view a lot of pages and items to locate the items they wish to buy, compare prices, or make the decision to purchase. If these URLs are being mined to find the most visited pages so items on them can be recommended, the system is likely going to suffer from serious scalability problems when generating personalized recommendations. The visited URLs can be used in finding top most rated items, best seller items and retailers in generating non-personalized recommendations.

Ways of reducing scalability problems such as reducing customer data on the system and alternative information for personalized recommendation generation other than mining web pages is being considered in this paper. The SEP framework is as shown in Figure 1.

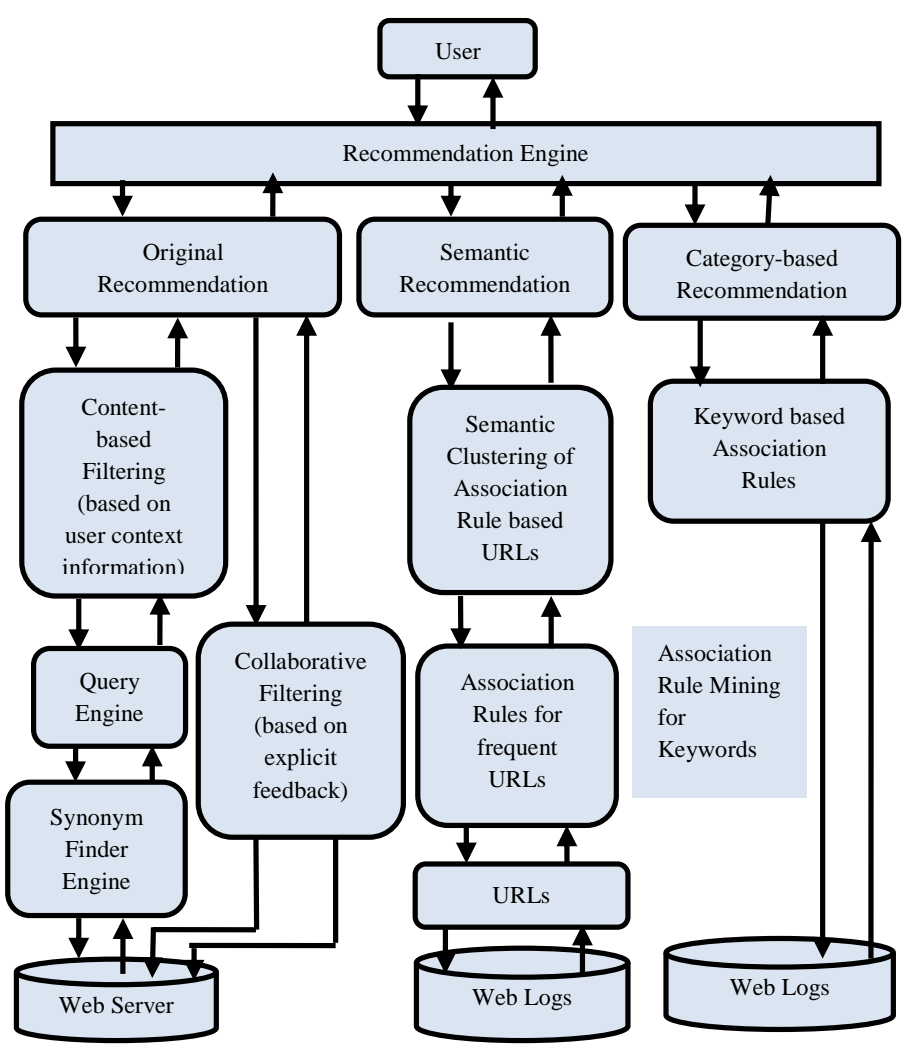

Figure 1:Semantic Enhanced Personalizer (SEP) Architecture (Sharma and Suman, 2011)

\section{PROPOSED MODEL}

The proposed hybrid item recommender model is as shown in Figure 2. The Figure shows user data being communicated to the recommendation engine (RE) of the online store, and the RE carrying out content-based, collaborative and demographic filtering on the data in other to generate recommendations. The recommendations generated are then communicated to the user via the user interface through the web server.

It is imperative to note that in the model, each category of recommendation will be generated depending on the availability of data for its processing. E.g. when shoppers provides demographic information on the store, demographic recommendations are generated. Consequently, the same goes for the content-based and collaborative recommendations when contents are specified and purchases are made. 


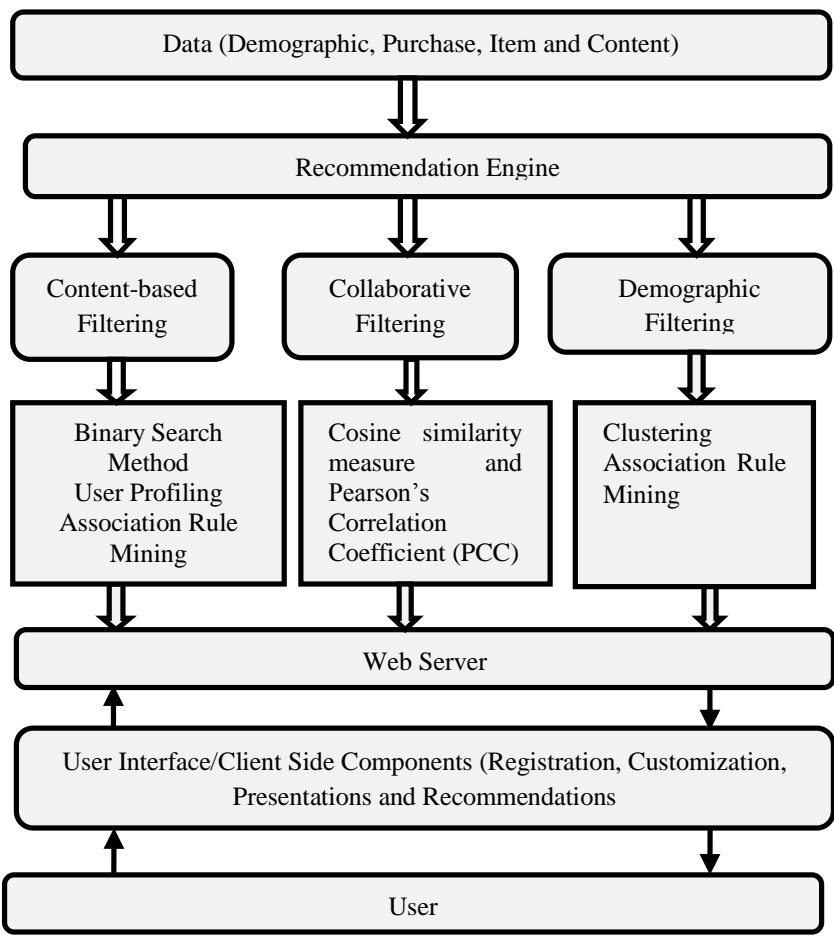

Figure 2: Proposed Model Architecture

Scalability concerns are addressed in the model by the algorithm only having to scale through content, purchase and demographic data. The model further addresses information overloading by displaying recommendations in mixed hybridization under html tags. The provision of demographic recommendations will address the cold-start/new-user problems so shoppers can get personalized item recommendations regardless of not having ratings or having sparse ratings on the system.

The proposed model formulation was carried out by substituting the ratings in the collaborative recommendation model for purchases. The content-based recommendation model was expressed mathematically for the search-based, user profiling and association rule mining processes. Same goes for the demographic recommendation model's clustering and association rule mining processes. The final hybrid item recommender model equation was expressed using an existing hybridization model equation for which the conditions for which the hybrid model holds were redefined in the context of this paper.

\subsection{Content-based Recommendations}

The model formulation for the content-based binary search expression, user profiling and the association rule mining expressions is as shown in Equation i, ii and iii. The final mathematical model for the content-based recommendation is expressed in Equation iv.

The binary search expression is as shown below in Equation i,

$\sum_{i=1}^{n} \underset{i}{R}\left(\boldsymbol{U}_{i}\right)=\left[\frac{\sum_{i=1}^{n} I_{i}\left(\boldsymbol{C}_{i}\right)}{2}-\left(\sum_{i=1}^{n} \boldsymbol{U}_{i}-\sum_{i=1}^{n} V_{i}\right)\right]$

where
$R$ represents a recommendation made by the system to a shopper;

$U$ represents a content specified by a shopper;

$V$ represents keyword extracted from a content specified;

$I$ represents an item on the system;

$C$ represents an item category on the system;

$\sum_{i=1}^{n} U$ represents the set of all search contents specified by shoppers;

$\sum_{i=1}^{n} V_{i}$ represents the set of all keywords extracted from search contents $\sum_{i=1}^{n} U$;

$\sum_{i=1}^{n} I_{i}$ represents the set of all items on the system;

$\sum_{i=1}^{n} \underset{i}{C}$ represents the set of all the categories of items on the system;

$\underset{i}{I}(\underset{i}{C})$ represents an items from a category of the system;

$\sum_{i=1}^{n} I_{i}\left(C_{i}\right)$ represents the set of all items from all categories of the system;

$\sum_{i=1}^{n} R_{i}\left(U_{i}\right)$ represents the set of all recommendations made from all specified contents;

$\frac{\sum_{i=1}^{n} I_{1}\left(\begin{array}{l}C \\ C\end{array}\right)}{2}$ represents all items from all categories of the store divided into two (2) parts (binary search); and

$\left(\sum_{i=1}^{n} U_{i}-\sum_{i=1}^{n} V_{i}\right) \quad$ represents the expression for the extraction of keywords from contents.

The user profiling expression is also as shown below in Equation ii,

$$
\sum_{i=1}^{n} \underset{i}{R}(\underset{i}{P})=\sum_{i=1}^{n} \underset{i}{I}(\underset{i}{C})-\left(\sum_{i=1}^{n} \underset{i}{H}-\sum_{i=1}^{n} \underset{i}{Q}\right)
$$

where

$I$ represents an item on the system; 
$C$ represents an item category on the system;

$R$ represents a recommendation made by the system to a shopper;

$\sum_{i=1}^{n} I_{i}(\underset{i}{C})$ represents the set of all items from all categories of the system;

$P$ represents a profile created by the system for a shopper;

$R(P)$ represents the recommendations made by the system for a profile;

$\sum_{i=1}^{n} R_{i}\left(P_{i}\right)$ represents the set of all recommendations made for all profiles by the system;

$H$ represents a transaction record stored in a profile for a shopper;

$\underset{i}{H}\left(P_{i}\right)$ represents a transaction record recorded in a profile by the system;

$\sum_{i=1}^{n} H_{i}$ represents the set of all transaction records of shoppers;

$Q$ represents a purchase made by a shopper;

$\sum_{i=1}^{n} \underset{i}{Q}$ represents the set of all purchases made by shoppers; $\left(\sum_{i=1}^{n} H_{i}-\sum_{i=1}^{n} \underset{i}{Q}\right)$ expresses as recommendations the remaining items in the categories of purchase after the items purchased has been extracted, this thus gave the expression.

Finally, the association rule mining expression is as shown below in Equation iii.

$$
R(\underset{i}{I} \underset{i}{Q})=>\sum_{i \in I}^{n} \underset{1}{Q}, \underset{2}{Q}, \underset{3}{Q}, \underset{4}{Q} \ldots \ldots \ldots . . Q_{n}
$$

where

$I$ represents an item on the system;

$Q$ represents a purchase made by a shopper;

$\sum_{i=1}^{n} \underset{i}{Q}$ represents the set of all purchases made by shoppers;and
$R(\underset{i}{I} \underset{i}{Q})$ represents recommended associated items $\underset{i}{I}$ for any purchased item $\underset{i}{Q}$;

The final expression for content-based recommendations (CBR) is given as shown in Equation iv

$$
C B R=\sum_{1}^{n} R\left(U_{i}\right)+\sum_{i}^{n} \underset{i}{R}(\underset{i}{P})+R(\underset{i}{I} \underset{i}{Q})
$$

\subsection{Collaborative Recommendations}

Equation $\mathrm{v}$ shows the expression for the cosine measure. The vector similarity/cosine measure was used to find the similarity between two shoppers using their purchase records. The cosine measure views two shoppers as vectors $a$ and $b$ in an n-dimensional space of items. If the shoppers' purchases are similar, they are assumed to point in the same direction in the item space. If their purchases are dissimilar, they are assumed to point in different directions in the item space. Similarity is then found for the shoppers by calculating the cosine of the angle between the vectors.

$$
\operatorname{Sim}(\vec{a}, \vec{b})=\cos \vec{a}, \vec{b}=\frac{\vec{a} \cdot \vec{b}}{\overline{\|a\|_{2} *\|\vec{b}\|_{2}}}
$$

The cosine measure however has the disadvantage of not finding how the individual shoppers' purchases deviate from the average purchases of the two shoppers. This disadvantage was addressed by the adjusted cosine measure otherwise referred to as the Pearson's Correlation Coefficient (PCC). The PCC was used to measure how much purchases by common shoppers for a pair of items deviated from average purchases for those items in the proposed model. It is as expressed in Equation vi.

$$
\begin{aligned}
& \operatorname{Sim}(a, b)= \\
& \sum_{u \subset \text { purchasedBoth }(a, b)}\left(r_{u a}-\overline{r_{a}}\right)\left(r_{u b}-\overline{r_{b}}\right) \\
& \sqrt{\sum_{u \subset \text { purchasedBoth }(a, b)\left(r_{u a}-\overline{r_{a}}\right)^{2}} \sqrt{\sum_{u \subset \text { purchasedBoth }(a, b)}\left(r_{u b}-\overline{r_{b}}\right)^{2}}}
\end{aligned}
$$

From Equation vi, $u \subset$ purchasedBoth $(a, b)$ is the set of all items purchased both by vectors $a$ and $b$ for which similarity is being found. ris the purchases made for which the sum of the purchases by common shoppers for a pair of items differ from the average purchases for the items. The final expression for collaborative recommendation (CR) is as shown in Equation vii.

$C R=\operatorname{Sim}(\vec{a}, \vec{b})+\operatorname{Sim}(a, b)$

\subsection{Demographic Recommendations}

The clustering expression and the association rule mining expression are as shown in Equations viii and ix. The final demographic recommendation expression is as shown in Equation $\mathrm{x}$.

The clustering expression is as shown in Equation viii,

$$
\sum_{i=1}^{n} R(\underset{i}{I} . \underset{i}{X})=\sum_{i=1}^{n} \underset{i}{I}(\underset{i}{S} . \underset{i}{D})
$$


$I$ represents an item on the system;

$D$ represents a demographic characteristic of shoppers;

$\sum_{i=1}^{n} D_{i}$ represents all the demographic characteristics of shoppers obtained by the system;

$X$ represents a demographic cluster;

$\sum_{i=1}^{n} X_{i}$ represents the set of all demographic clusters formed;

$S$ represents a shopper on the system;

$\sum_{i=1}^{n} S_{i}$ represents all shoppers on the system;

$\underset{i}{S . D_{i}}$ represents a shopper with a demographic characteristic;

$\sum_{i=1}^{n} \underset{i=1}{I}\left(S_{i} . D_{i}\right)$ represents the items associated to shoppers' demographics;

Adding shoppers to predefined clusters thus gives

$$
\left(\sum_{i=1}^{n} \underset{i}{X}=\sum_{i=1}^{n} \underset{1}{S} . \underset{1}{D}+\sum_{i=2}^{n} \underset{2}{S} . \underset{2}{D}+\ldots .+\sum_{i=n}^{n} \underset{n}{S} . \underset{n}{D}\right)_{;}
$$

$R\left(I_{i} . \underset{i}{X}\right)$ represents the recommendation of items made to a demographic cluster; and

$\sum_{i=1}^{n} R\left(I_{i} . \underset{i}{X}\right)$ represents the recommendation of items made to all demographic clusters.

Items have been associated to the clusters (association rule mining) as shown in Equation ix,

$$
\sum_{i=1}^{n} R\left(I_{i} \underset{i}{X}\right)=>\sum_{i=1}^{n} I_{i}
$$

where

$I$ represents an item on the system;

$X$ represents a demographic cluster;

$\sum_{i=1}^{n} I_{i}$ represents the set of all items on the system; and

$\sum_{i=1}^{n} R\left(I_{i} . X{ }_{i}\right)$ represents the recommendation of items made to all demographic clusters.

The final expression for demographic recommendations (DR) is given as

$$
D R=\sum_{i=1}^{n} R\left(I_{i} \cdot X_{i}\right)+\sum_{i=1}^{n} R\left(I_{i} \underset{i}{X}\right)
$$

The proposed hybrid item recommendation model for the content-based, collaborative and demographic recommendations has been hybridized using the hybridization expression of [3]. The hybridized expression is given as shown in Equation xi.

$$
R_{u, i}=\alpha D R_{u, i}+\beta C B R_{u, i}+\gamma C R_{u, i}
$$

From Equation xi, 'DR' represents demographic recommendations, 'CR' represents collaborative recommendations, and 'CBR' represents content-based recommendations respectively. These recommendations were combined by [3] into a single hybridized model using confidence values $\alpha, \beta$, and $\gamma$ which represents a sort of confidence given to each of the recommendations.

According to [3], the greater the number of user ratings available to the collaborative filter, the greater the confidence given to the collaborative recommendation process of the system. Likewise, the greater the item ratings available to the content-based filter, the greater the confidence given to the content-based recommendation process of the system. Finally, the greater the number of users with no ratings for which demographic similarities are being found, the greater the confidence given to the demographic recommendation process of the system. The values of $\alpha, \beta$, and $\gamma$ changes dynamically depending on the available ratings expressed by shoppers.

For the proposed model, $\alpha, \beta$, and $\gamma$ also represent the confidence given to each of the recommendations. For the demographic recommendations, the greater the number of demographic characteristics used in finding user similarities in the demographic filtering process, the stronger the demographic filter, and thus the better the quality of the demographic recommendations provided by the system. $\beta$ represents the confidence given to the content-based recommendation process, which implies that, the greater the number of items in the database for which the system can compare active shopper's purchases and contents with, the greater the strength given to the content-based filter, and the better the content-based recommendations provided.

Furthermore, $\gamma$ represents the confidence given to the collaborative recommendations process, which implies, the greater the similarity of an active to other similar shoppers, the greater the strength given to the collaborative filter and the better the quality of the collaborative recommendations provided by the system.

\subsection{Proposed Model's Implementation}

The proposed model was implemented using web development tools into a workable system. The system is an online store that provides content-based, collaborative and demographic item recommendations (personalized and nonpersonalized). The system is embedded with a recommendation engine that generates these recommendations. The recommendations are generated concurrently by the system and are presented in a mixed hybridization style on the store. The system presents its recommendations to shoppers and visitors via the homepage of the store. These recommendations are generated by the system as data required by each of the filtering processes to produce these recommendations are communicated to the 
filters. The new system has been christened 'Passions Stores'. For content-based recommendation, the system first extracts all keywords from search contents specified by shoppers to the system and proceeds to divide the entire sorted list of items is in two (2) parts. Then the input element (item/items being sought for) is compared with the mid element. The system then tries to find if the sought item(s) is from the left or the right side of the list based on a conquer and divide strategy. If the item(s) sought for is found, it is returned as recommendations. The system also creates profiles for all shoppers and adds their purchase information to it. From items purchased by shoppers, the system checks for other items in the categories of their purchase and presents them as recommendations. Lastly, the system finds items often purchased alongside the items in the purchase records that have not been considered and presents them as recommendations.

For the collaborative recommendations, the system first retrieves all purchases made by shoppers from their profiles and finds similarities in the purchases. If similarities are found for a pair of shoppers, the system recommends the items considered by each of the shoppers not yet considered by the other. The condition for similarity between two shoppers has been set to be two items.

For the demographic recommendations, all items in the database was assigned a gender character which can either be 'male', 'female' or 'general' (unisex). Demographic clusters age ('11-20', '21-30', '31-40', '41-50', and '50 and above'), gender ('male' and 'female'), and religion ('Christianity' and 'Islam') were formed and shoppers were added to these clusters based on the demographic information they provided. The system then assigns item and item categories to these clusters for recommendations.

Figure 3 shows the homepage of the Passions system which shows content-based recommendations being presented under the tag "Here are the items similar to your past purchases", collaborative recommendations being presented under the tag "Find out what other shoppers are buying", and demographic recommendations being presented under the heading "Here are the items we believe might suit you". These recommendations are generated for each shopper upon communication of data (through interactions) required for its processing to the system by the shopper.

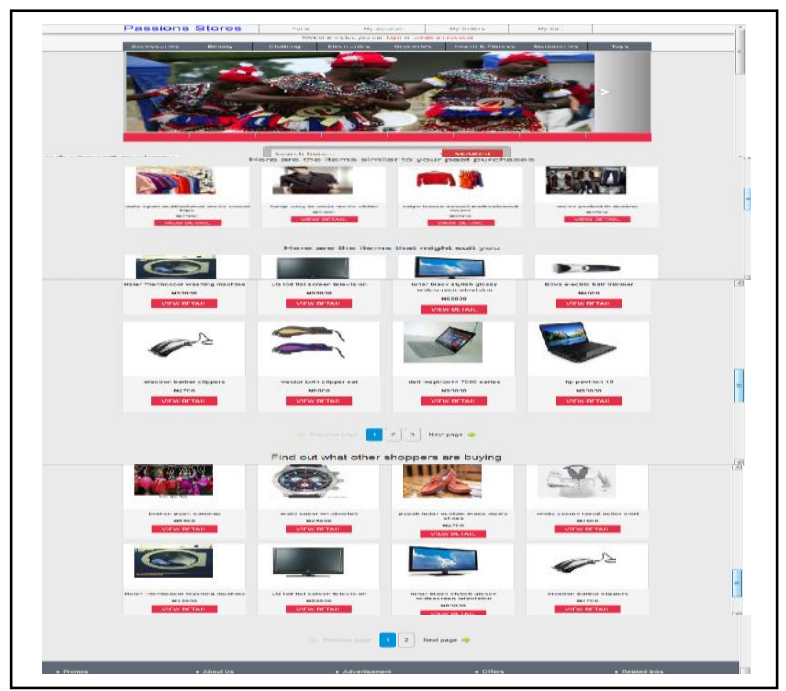

Figure 3: Items Recommendation Page on Passions Store

\section{SYSTEM EVALUATION}

Two metrics were used in evaluating the performance of the Passions. This metrics are used in measuring prediction/recommendation ability and accuracy of a recommender system. The metrics are the Mean Absolute Error (MAE) and the Root Mean Square Error (RMSE). The MAE is a quantity used to measure how close forecasts or predictions are to the eventual outcomes. It is a common measure of forecast error. It gives the average of the absolute values for the errors in a set of recommendations. It If $\gamma^{\prime}$ is a vector of $\mathrm{n}$ predicted values and $\gamma$ is the vector of actual values corresponding to the function which generated the recommendations, then the Mean Absolute Error can be estimated by the following expression depicted in Equation xii.

$$
M A E=\frac{1}{n} \sum_{i=1}^{n}\left(\gamma-\gamma^{\prime}\right)
$$

The Root Mean Square Error is the sample standard deviation of differences between predicted values and observed values. It is a quadratic scoring rule which measures the magnitude of the errors. It is used for calculating larger absolute errors and is a good measure of accuracy. It can be estimated by the following expression depicted in Equation xiii.

$$
R M S E=\sqrt{\text { MeanAbsoluteError }}=\sqrt{\frac{1}{n} \sum_{i=1}^{n}\left(\gamma-\gamma^{\prime}\right)}(\text { xiii })
$$

These metrics are often used together in diagnosing error variations in a set of recommendations. The RMSE value is always greater than the MAE value. The greater the difference between MAE and RMSE values, the greater the variance in the individual errors in the recommendations. But if the RMSE value is equal to the MAE value, then all the errors in the recommendation are of the same magnitude.

Recommendations of the Passions system were evaluated by comparing it with the recommendations provided by the Jumia online store (a Nigerian online store implemented similar to online stores abroad, i.e. using with foreign template). The evaluation was performed using user studies method. A hypothesis was formed that the Passions system provides better recommendations than the Jumia system store. The results of the experiment showed that the total MAE value of the Passions system was smaller than that of the Jumia store for content-based and collaborative recommendations.

The same applies to the RMSE values. This implied that there were lesser errors in the recommendations provided by the Passions system compared to the Jumia online store, and that the Passions system was more accurate in its recommendations. The results are as computed below. No comparison was made for the demographic recommendations as the Jumia system does not provide demographic recommendations. Table 1, Table 2, Table 3, Table 4 and Table 5 present the results of the evaluation performed on the Passions system (new system) and the Jumia system. Table 6, Table 7, Table 8, and Table 9 further presents the MAE and RMSE values' comparisons for both systems. The results for the MAE and RMSE values shown in Table 6, Table 7, Table 8 , and table 9 has been further depicted graphically as shown in Figure 4, Figure 5, Figure 6 and Figure 7.

Errors in recommendations cannot be eliminated but can be reduced. The errors of the existing system personalized content-based and collaborative recommendations have been 
reduced by the Passions system. The error rates of the Passions system has been expressed in percentages of the error rates of the Jumia system are as follows:

\section{A. Content-based Recommendation MAE values}

Total MAE value $($ Jumia $)=4.9$ and Total MAE

value $($ Passions $)=1.9$

Thus, percentage MAE value of Passions in comparison to Jumia $=$

$$
\frac{1.9}{4.9} * 100=38.76 \%
$$

Thus the percentage MAE value of Passions system is $38.76 \%$ of the Jumia system showing $61.24 \%$ MAE reduction and it implies better performance of the Passions system.

B. Content-based Recommendation RMSE values

RMSE value $($ Jumia $)=2.2136$ and RMSE value

$($ Passions $)=1.3784$

Thus, percentage RMSE value of Passions system in comparison to Jumia $=$

$$
\frac{1.3784}{2.2136} * 100=62.27 \%
$$

Thus the percentage RMSE value of Passions system is $62.27 \%$ of the Jumia system showing $37.23 \%$ RMSE reduction and it implies better performance of the Passions system.

C. Collaborative Recommendation MAE values

Total MAE value $($ Jumia $)=19$ and Total MAE

value $($ Passions $)=7$

Thus, percentage MAE value of Passions in comparison

$$
\frac{7}{19} * 100=36.84 \%
$$

Thus the percentage MAE value of Passions system is $36.84 \%$ of the Jumia system showing $63.16 \%$ MAE reduction and it implies better performance of the Passions system.

D. Collaborative Recommendation RMSE values RMSE value $($ Jumia $)=4.3589$ and RMSE value $($ Passions $)=2.6458$

Thus, percentage RMSE value of Passions in comparison to Jumia $=$

$$
\frac{2.6458}{4.3589} * 100=60.70 \%
$$

Thus the percentage RMSE value of Passions system is $60.70 \%$ of the Jumia system. This shows $39.30 \%$ RMSE error reduction and it implies better performance of the Passions system.
Table 1: Content-based Recommendations Mean Absolute Error (MAE) and Root Mean Square Error (RMSE) values of the Passions System

\begin{tabular}{|l|l|l|l|c|c|c|}
\hline \multicolumn{7}{|c|}{ Content-based Recommendations } \\
\hline $\begin{array}{l}\text { Shop- } \\
\text { pers }\end{array}$ & $\begin{array}{l}\text { No. of } \\
\text { Items } \\
\text { Purch- } \\
\text { ased }\end{array}$ & $\begin{array}{l}\text { Actual } \\
\text { Rec. } \\
\text { (A) }\end{array}$ & $\begin{array}{l}\text { Predicted } \\
\text { Rec. (P) }\end{array}$ & $\begin{array}{l}\text { Error } \\
\text { = A-P }\end{array}$ & $\begin{array}{l}\text { ABS } \\
\text { (A-P) }\end{array}$ & MAE \\
\hline 1 & 4 & 24 & 21 & -3 & 3 & 0.3 \\
\hline 2 & 5 & 29 & 30 & -1 & 1 & 0.1 \\
\hline 3 & 2 & 30 & 33 & -3 & 3 & 0.3 \\
\hline 4 & 5 & 39 & 37 & 2 & 2 & 0.2 \\
\hline 5 & 4 & 26 & 27 & 1 & 1 & 0.1 \\
\hline 6 & 7 & 38 & 38 & 3 & 3 & 0.3 \\
\hline 7 & 4 & 27 & 25 & 2 & 2 & 0.2 \\
\hline 8 & 6 & 45 & 46 & 1 & 1 & 0.1 \\
\hline 9 & 4 & 30 & 31 & 2 & 1 & 0.2 \\
\hline 10 & 6 & 26 & 25 & 1 & 1 & 0.1 \\
& & & & & & \\
\hline & & & & & & \\
\hline
\end{tabular}

\begin{tabular}{|c|c|c|c|c|c|c|}
\hline & \multicolumn{6}{|c|}{ Collaborative Recommendations } \\
\hline $\begin{array}{l}\text { Shop- } \\
\text { pers }\end{array}$ & $\begin{array}{l}\text { No. of } \\
\text { Similar } \\
\text { Shoppers }\end{array}$ & $\begin{array}{l}\text { Actual } \\
\text { Rec. (A) }\end{array}$ & $\begin{array}{l}\text { Predicted } \\
\text { Rec. (P) }\end{array}$ & $\begin{array}{l}\text { Error } \\
=\mathrm{A}-\mathrm{P}\end{array}$ & $\begin{array}{l}\text { ABS } \\
\text { (A-P) }\end{array}$ & MAE \\
\hline 1 & 2 & 28 & 37 & -9 & 9 & 0.9 \\
\hline 2 & 3 & 45 & 40 & 5 & 5 & 0.5 \\
\hline 3 & 3 & 49 & 56 & -7 & 7 & 0.7 \\
\hline 4 & 1 & 15 & 20 & -5 & 5 & 0.5 \\
\hline 5 & 2 & 30 & 35 & -5 & 5 & 0.5 \\
\hline 6 & 3 & 34 & 25 & 9 & 9 & 0.9 \\
\hline 7 & 3 & 28 & 35 & -7 & 7 & 0.7 \\
\hline 8 & 4 & 32 & 41 & -9 & 9 & 0.9 \\
\hline 9 & 5 & 40 & 37 & 3 & 3 & 0.3 \\
\hline \multirow[t]{3}{*}{10} & 3 & 27 & 16 & 11 & 11 & 0.11 \\
\hline & & & & & \multicolumn{2}{|c|}{ MAE total $=7$} \\
\hline & & & & \multicolumn{3}{|c|}{$\mathbf{R M S E}=2.645751311$} \\
\hline
\end{tabular}

Table 2: Collaborative Recommendations Mean Absolute Error (MAE) and Root Mean Square Error (RMSE) values of the Passions System 
Table 3: Demographic Recommendations Mean Absolute Error (MAE) and Root Mean Square Error (RMSE) values of the Passions System

\begin{tabular}{|c|c|c|c|c|c|c|}
\hline \multicolumn{7}{|c|}{ Demographic Recommendations } \\
\hline $\begin{array}{l}\text { Sho- } \\
\text { ppers }\end{array}$ & $\begin{array}{l}\text { Demograp } \\
\text {-hics Used }\end{array}$ & $\begin{array}{l}\text { Actual } \\
\text { Rec. (A) }\end{array}$ & $\begin{array}{l}\text { Predicted } \\
\text { Rec. (P) }\end{array}$ & $\begin{array}{l}\text { Error } \\
=\mathrm{A}-\mathrm{P}\end{array}$ & $\begin{array}{l}\text { ABS } \\
\text { (A-P) }\end{array}$ & MAE \\
\hline 1 & $\begin{array}{l}\text { Gender, } \\
\text { Age, } \\
\text { Religion }\end{array}$ & 72 & 74 & -2 & 2 & 0.2 \\
\hline 2 & $\begin{array}{l}\text { Gender, } \\
\text { Age, } \\
\text { Religion }\end{array}$ & 57 & 60 & -3 & 3 & 0.3 \\
\hline 3 & $\begin{array}{l}\text { Gender, } \\
\text { Age, } \\
\text { Religion }\end{array}$ & 68 & 70 & -2 & 2 & 0.2 \\
\hline 4 & $\begin{array}{l}\text { Gender, } \\
\text { Age, } \\
\text { Religion }\end{array}$ & 57 & 60 & -3 & 3 & 0.3 \\
\hline 5 & $\begin{array}{l}\text { Gender, } \\
\text { Age, } \\
\text { Religion }\end{array}$ & 70 & 72 & -2 & 2 & 0.2 \\
\hline 6 & $\begin{array}{l}\text { Gender, } \\
\text { Age, } \\
\text { Religion }\end{array}$ & 56 & 58 & -2 & 2 & 0.2 \\
\hline 7 & $\begin{array}{l}\text { Gender, } \\
\text { Age, } \\
\text { Religion }\end{array}$ & 68 & 71 & -3 & 3 & 0.3 \\
\hline 8 & $\begin{array}{l}\text { Gender, } \\
\text { Age, } \\
\text { Religion }\end{array}$ & 64 & 67 & -3 & 3 & 0.3 \\
\hline 9 & $\begin{array}{l}\text { Gender, } \\
\text { Age, } \\
\text { Religion }\end{array}$ & 62 & 65 & -3 & 3 & 0.3 \\
\hline 10 & $\begin{array}{l}\text { Gender, } \\
\text { Age, } \\
\text { Religion }\end{array}$ & 53 & 55 & -2 & 2 & 0.2 \\
\hline & & & & RMS & $\begin{array}{l}\text { E tota } \\
=1.581\end{array}$ & \\
\hline
\end{tabular}

Table 4: Content-based Recommendations Mean Absolute Error (MAE) and Root Mean Square Error (RMSE) values of the Jumia System

\begin{tabular}{|l|c|c|c|c|c|}
\hline \multicolumn{7}{|c|}{ Content-based Recommendations } \\
\hline Items Rated & $\begin{array}{l}\text { Actual } \\
\text { Rec. (A) }\end{array}$ & $\begin{array}{l}\text { Predicted } \\
\text { Rec. (P) }\end{array}$ & $\begin{array}{l}\text { Error } \\
\text { = A-P }\end{array}$ & $\begin{array}{l}\text { ABS } \\
\text { (A-P) }\end{array}$ & MAE \\
\hline 3 Perfumes & 13 & 15 & -2 & 2 & 0.2 \\
\hline $\begin{array}{l}\text { 2 Android } \\
\text { Phones }\end{array}$ & 35 & 40 & -5 & 5 & 0.9 \\
\hline 6 Makeup boxes & 30 & 36 & -6 & 6 & 0.6 \\
\hline 5 TV sets & 24 & 20 & 4 & 4 & 0.4 \\
\hline 3 Wristwatches & 9 & 6 & 3 & 3 & 0.3 \\
\hline 5 Printers & 12 & 8 & 6 & 6 & 0.6 \\
\hline 2 Shoes & 18 & 10 & 8 & 8 & 0.8 \\
\hline 3 Handbags & 30 & 25 & 5 & 5 & 0.5 \\
\hline 2 Gas cookers & 8 & 7 & 1 & 1 & 0.1 \\
\hline 7 Dresses & 15 & 23 & -8 & 8 & 0.8 \\
\hline & & & & & \\
\hline & & \multicolumn{7}{|c|}{ MAE total = 4.9} \\
& & & & & \\
\hline
\end{tabular}

Table 5: Collaborative Recommendations Mean Absolute Error (MAE) and Root Mean Square Error (RMSE) values of the Jumia System

\begin{tabular}{|l|c|c|c|c|c|}
\hline \multicolumn{6}{|c|}{ Collaborative Recommendations } \\
\hline $\begin{array}{l}\text { Items } \\
\text { Rated }\end{array}$ & $\begin{array}{l}\text { Actual } \\
\text { Rec. } \\
\text { (A) }\end{array}$ & $\begin{array}{l}\text { Predicted } \\
\text { Rec. (P) }\end{array}$ & $\begin{array}{l}\text { Error } \\
\text { A-P }\end{array}$ & ABS (A-P) & MAE \\
\hline $\begin{array}{l}\text { 2 BB } \\
\text { Phones }\end{array}$ & 7 & 30 & -23 & 23 & 2.3 \\
\hline $\begin{array}{l}\text { 2 Android } \\
\text { Phones }\end{array}$ & 6 & 25 & -19 & 19 & 1.9 \\
\hline 3 Belts & 3 & 15 & -12 & 12 & 1.2 \\
\hline 4 Dresses & 5 & 30 & -25 & 25 & 2.5 \\
\hline $\begin{array}{l}\text { 2 Washing } \\
\text { Machines }\end{array}$ & 8 & 20 & -12 & 12 & 1.2 \\
\hline 3 Laptops & 4 & 40 & -36 & 36 & 3.6 \\
\hline 3 Shoes & 6 & 15 & -9 & 9 & 0.9 \\
\hline 4 Sandals & 9 & 25 & -16 & 16 & 1.6 \\
\hline 2 Handbags & 7 & 30 & -23 & 23 & 2.3 \\
\hline 3 Shirts & 5 & 20 & -15 & 15 & 1.5 \\
\hline & & & & & \\
\hline & & & & & \\
& & & & & \\
\hline
\end{tabular}

Table 6: MAE values comparison for Content-based Recommendations on the Jumia System and the Passions System

Content-based Recommendations

\begin{tabular}{|c|c|c|}
\hline Shoppers & MAE Jumia System & MAE Passions System \\
\hline 1 & 0.2 & 0.3 \\
\hline 2 & 0.5 & 0.1 \\
\hline 3 & 0.6 & 0.3 \\
\hline 4 & 0.4 & 0.2 \\
\hline 5 & 0.3 & 0.1 \\
\hline 6 & 0.7 & 0.3 \\
\hline 7 & 0.8 & 0.2 \\
\hline 8 & 0.5 & 0.1 \\
\hline 9 & 0.1 & 0.2 \\
\hline 10 & 0.8 & 0.1 \\
\hline
\end{tabular}


Table 7: RMSE values comparison for Content-based Recommendations on the Jumia System and the Passions System

\begin{tabular}{|c|c|c|}
\hline \multicolumn{3}{|c|}{ Content-based Recommendations } \\
\hline Shoppers & $\begin{array}{c}\text { RMSE Jumia } \\
\text { System }\end{array}$ & RMSE Passions System \\
\hline 1 & 2.213594362 & 1.140175425 \\
\hline 2 & 2.213594362 & 1.140175425 \\
\hline 3 & 2.213594362 & 1.140175425 \\
\hline 4 & 2.213594362 & 1.140175425 \\
\hline 5 & 2.213594362 & 1.140175425 \\
\hline 6 & 2.213594362 & 1.140175425 \\
\hline 7 & 2.213594362 & 1.140175425 \\
\hline 8 & 2.213594362 & 1.140175425 \\
\hline 9 & 2.213594362 & 1.140175425 \\
\hline 10 & 2.213594362 & \\
\hline & & \\
\hline
\end{tabular}

Table 8: MAE values comparison for Collaborative Recommendations on the Jumia System and the Passions System

\begin{tabular}{|c|c|c|}
\hline \multicolumn{3}{|c|}{ Collaborative Recommendations } \\
\hline Shoppers & MAE Jumia System & MAE Passions System \\
\hline 1 & 2.3 & 0.9 \\
\hline 2 & 1.9 & 0.5 \\
\hline 3 & 1.2 & 0.7 \\
\hline 4 & 2.5 & 0.5 \\
\hline 5 & 1.2 & 0.5 \\
\hline 6 & 3.6 & 0.9 \\
\hline 7 & 0.9 & 0.7 \\
\hline 8 & 1.6 & 0.9 \\
\hline 9 & 2.3 & 0.3 \\
\hline 10 & 1.5 & 1.1 \\
\hline
\end{tabular}

Table 9: RMSE values comparison for Collaborative Recommendations on the Jumia System and the Passions System

\begin{tabular}{|c|c|c|}
\hline \multicolumn{3}{|c|}{ Collaborative Recommendations } \\
\hline Shoppers & $\begin{array}{c}\text { RMSE Jumia } \\
\text { System }\end{array}$ & RMSE Passions System \\
\hline 1 & 4.35889894 & 2.6457511311 \\
\hline 2 & 4.35889894 & 2.6457511311 \\
\hline 3 & 4.35889894 & 2.6457511311 \\
\hline 4 & 4.35889894 & 2.6457511311 \\
\hline 5 & 4.35889894 & 2.6457511311 \\
\hline 6 & 4.35889894 & 2.6457511311 \\
\hline 7 & 4.35889894 & 2.6457511311 \\
\hline 8 & 4.35889894 & 2.6457511311 \\
\hline 9 & 4.35889894 & 2.6457511311 \\
\hline 10 & 4.35889894 & 2.6457511311 \\
\hline
\end{tabular}

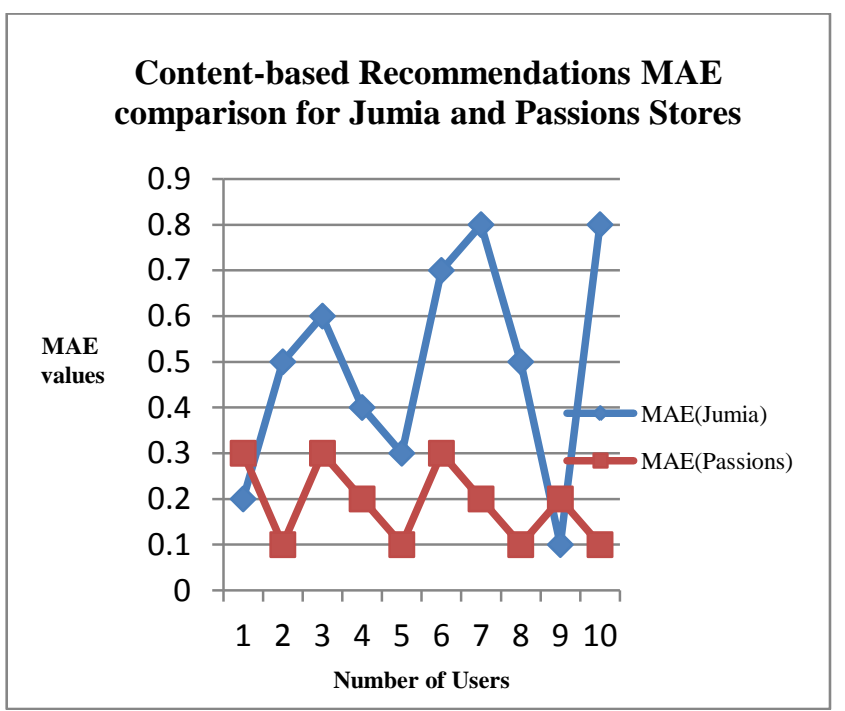

Figure 4: Graphical representation of MAE values comparison for Jumia System and the Passions System against the number of users in Content-based Recommendations 


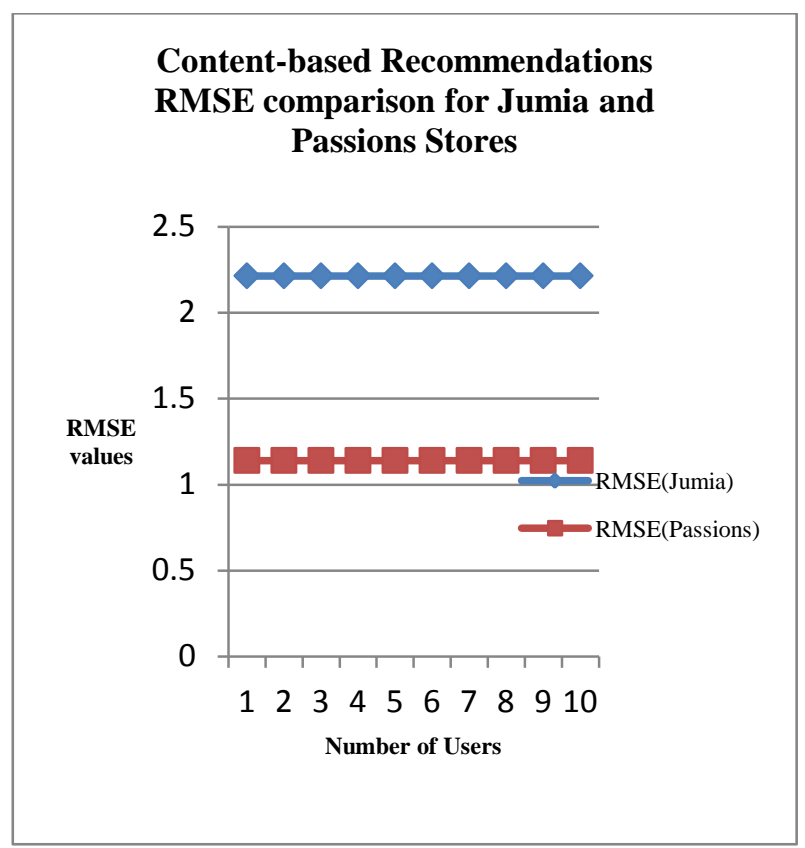

Figure 5: Graphical representation of RMSE values for Jumia System and the Passions System against the number of users in Content-based Recommendations

\section{Collaborative Recommendations MAE comparison for Jumia and Passions Stores}

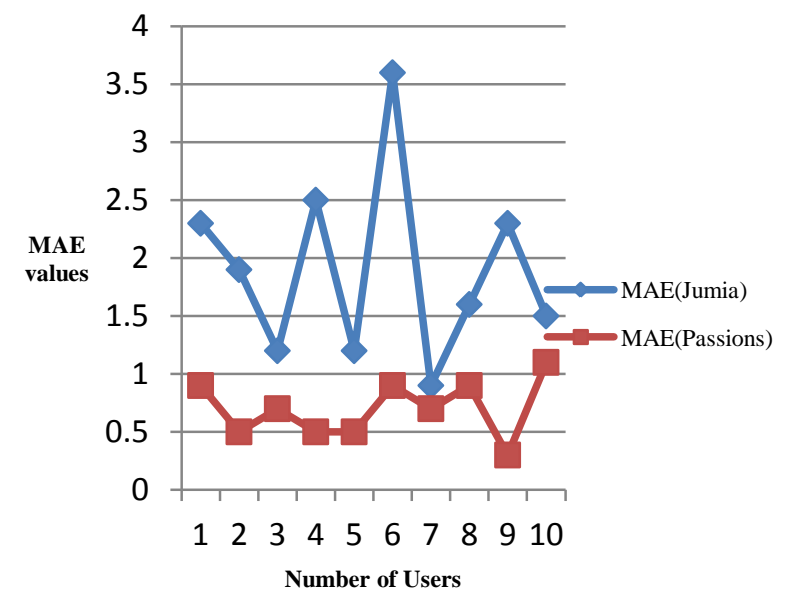

Figure 6: Graphical representation of MAE values for Jumia System and the Passions System against the number of users in Collaborative Recommendations
Collaborative Recommendations RMSE comparison for Jumia and Passions Stores

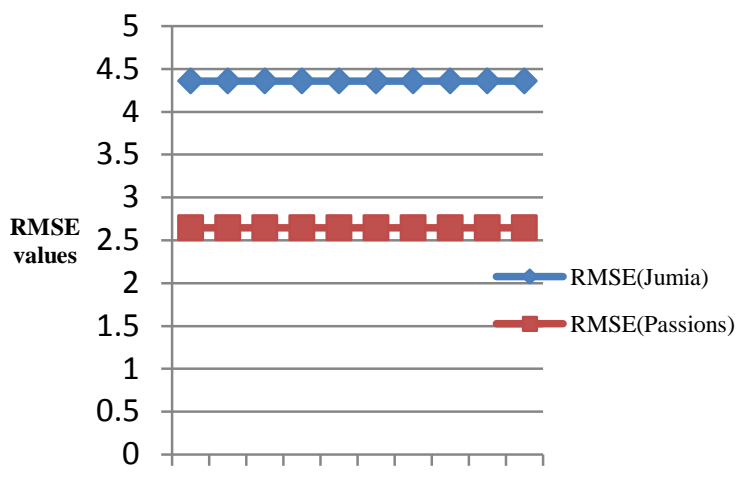

$\begin{array}{llllllllll}1 & 2 & 3 & 4 & 5 & 6 & 7 & 8 & 9 & 10\end{array}$

Number of Users

Figure 7: Graphical representation of RMSE values for Jumia System and the Passions System against the number of users in Collaborative Recommendations

\section{REFERENCES}

[1] Bobadilla, J., Ortega, F. and Hernando, A. 2012. A Collaborative Filtering Similarity Measure based on Singularities. Journal of Information Processing and Management, 48(2): pp. 204-217.

[2] Chen, T., and He, L. 2009. Collaborative Filtering based on Demographic Attribute Vector. In International Conference on Future Computer and Communication (FCC'09) (pp.225-229), Canada.

[3] Chikhaoui, B., Chiazzaro, M. and Wang, S. 2011. An Improved Hybrid Recommender System by Combining Predictions. In Advanced Information Networking and Applications (WAINA) 2011 IEEE Workshops (pp. 644649), Biopolis.

[4] Chu, W. and Park, S. T. 2009. Personalized Recommendations on Dynamic Content Using Predictive Bilinear Models. In Proceedings of the 18th International Conference on World Wide Web (pp. 691-700), Madrid, Spain.

[5] Deepa, R. and Hamsaveni, R. 2010. Online Customer Value Identification Based on Site Usage Time through Data Mining Analysis. Global Journal of Computer Science and Technology, 10(2): pp. 10-16.

[6] Deshpande, M. and Karypis, G. 2004. Item-based Top-N Recommendation Algorithms. ACM Transactions on Information Systems (TOIS), 22(1): pp. 143-177.

[7] Edson B., Santos, J., Marecelo, G. M. and Rudinei, C.2015. Evaluating the Impact of Demographic Data on a Hybrid Recommender Model. IADIS International Journal on WWW/Internet, 12(8): pp. 14-21.

[8] Escriche, M. and Symeon, P. 2011. User Profiling and Personalization Tools. 'WeKnowIt' Emerging, Collective Intelligence for Personal, Organizational, and Social Use Journal, 1(2): pp. 1-26. 
[9] Jafari, M., Sabzchi, F. S. and Irani, A. J. 2014. Applying Web Usage Mining Techniques to Design Effective Web Recommendation Systems: A Case Study. Journal of Advances in Computer Science, 3(2): pp. 78-90.

[10] Kabore, S. C. 2012. Design and Implementation of a Recommender System as a Module for Liferay Portal. In Barcelona School of Computing (FIB), University Polytechnic of Catalunya (UPC) (pp. 1-127), Barcelona, Spain.

[11] Mabude, C., N. 2014. Development of an Improved Model for Expertise Recommendation in Academic Research. Unpublished M.Sc Thesis Submitted to the Department of Computer Science and Engineering, Obafemi Awolowo University, Ile-Ife. 2014; pp. 1-129.

[12] Prassad, R. and Kumari, V. V. 2012. A Categorical Review of Recommender Systems. International Journal of Distributed and Parallel Systems (IJDPS), 3(5): pp. 73-83.

[13] Ristoski, P., Mencía, E. L. and Paulheim, H. 2014. A Hybrid Multi-Strategy Recommender System Using
Linked Open Data. Semantic Web Evaluation Challenge, 1(2): pp. 150-156.

[14] Safoury, L. and Salah, A. 2013. Exploiting User Demographic Attributes for Solving Cold-Start Problem in Recommender System. Lecture Notes on Software Engineering, 1(3): pp. 303-307.

[15] Sharma, S. K. and Suman, U. 2011. Design and Implementation of Architectural Framework of Recommender System for E-commerce. International Journal of Computer Science and Information Technology \& Security (IJCSITS), 1(2): pp. 153-162.

[16] Vozalis, M. and Margaritis, K. G. 2004. Enhancing Collaborative Filtering with Demographic Data: The Case of Item-based Filtering. In Proceedings of the Fourth IEEE International Conference on Intelligent Systems Designs and Application (pp. 1-6), Brazil.

[17] Zenebe, A., Ozok, A. and Norcio, A. F. 2005. Personalized Recommender Systems in E-Commerce and M-Commerce: A Comparative Study. Research Gate publications, 1(2): pp. 1-10. 